\title{
The Correction of Nonuniform Signal Intensity Profiles in Magnetic Resonance Imaging
}

\author{
John Listerud, Robert E. Lenkinski, Herbert Y. Kressel, and Leon Axel
}

\begin{abstract}
The increasing use of digital image data in Radiology has opened the door to the routine use of numerical image-enhancement techniques. Of course, numerical image processing cannot put information into the image which is not already there. However, if some means can be found to separate diagnostic image information from noise or artifact, the diagnostic information can be extracted with post-processing. The diagnostic quality of an exam may be enhanced by such numerical manipulations, even though technically, the information content of the digitized image is reduced.

1989 by W.B. Saunders Company
\end{abstract}

KEY WORDS: Magnetic resonance imaging, signalto-noise ratio, surface coil.

$\mathbf{T}$ HIS paper considers a common problem in magnetic resonance imaging (MRI), the nonuniform response of the receiver coil which results in shading of the image. In the case of the body or head coil, such a nonuniform response is a minor nuisance that may not be appreciated on casual visual inspection. In the case of the surface coil, nonuniform response is often used to localize signal reception, but its use unavoidably and severely shades intensity. Appropriate thresholding of a surface-coil image for one region, a lumbar spinal cord for example, often results in "blackening" or "whitening" of the regions far and near the coil, respectively. When diagnostic attention is confined to a small region, this provides an adequate exam. However, when interest is not confined to a small region, the digital image thresholds must be repeatedly adjusted to assess the entire image. Conceptually, the image contains two types of information,

From the Pendergrass Diagnostic Radiology Research Laboratory, University of Pennsylvania School of Medicine, Philadelphia and the David W. Devon Medical Imaging Center, Hospital of the University of Pennsylvania, Philadelphia.

Address reprint requests to John Listerud, MD, PhD, Pendergrass Diagnostic Radiology Research Laboratory, 36 th and Hamilton Walk, University of Pennsylvania, Philadelphia, PA 19104.

(C) 1989 by W.B. Saunders Company

0897-1889/89/0201-0010\$03.00/0 the surface coil response, and the anatomic intensity variation. Clearly the surface-coil response has no diagnostic relevance, and its removal does not degrade the diagnostic quality of the image.

In a working clinical environment, the images are not reviewed in digital format on the monitor, but after photography. Correction of an image by removal of the shading due to surface coil response will allow the entire image to be viewed with a single-threshold setting. Thus, more anatomic information survives photography in a corrected image, so that from a practical point of view, surface-coil correction is a desirable manipulation of digital image data. Shading in conventional body coil or head coil images is usually not so severe as to cause a problem on photography. However, three dimensional displays of serial sections, or stacked two dimensional data often use intensity thresholding to distinguish between different materials. Slight variations in intensity due to shading can hamper the ability of such techniques to distinguish between materials that are not widely separated by anatomic contrast.

Several authors ${ }^{1-3}$ have discussed an approach to correction that is motivated by the model of pixel intensity in a surface coil image as given by the "true" signal intensity, which represents anatomic and diagnostic information, weighted by the local surface coil respunse factor. If we can extract a map of the local surface coil response from the image, we may correct the image by dividing each pixel by the local response factor. This reduces the correction problem to the search for an efficient method for extracting the surface coil response map.

In an uncorrected surface coil image, the signal-to-noise ratio (SNR) varies across the extent of the image because the signal drops off with distance away from the coil, while the noise level remains constant. In the corrected image, both signal and noise have been rescaled to make signal approximately constant across the image while noise is unavoidably scaled up in those regions of low intensity. Theoretically, however, 
the SNR remains locally constant with respect to correction.

Consider a generic circular surface coil of radius $R$. We may make the simple observation that the surface coil response varies slowly over a distance of about $R / 2$. If the generic surface coil image is taken to have a field of view (FOV) of $2 \mathrm{R}$, then the coil response can be considered to be almost constant over a distance equal to FOV $/ 4$. The anatomic information varies more over this same distance. Stated more abstractly, these two types of information, coil response and anatomy, can be distinguished by the separation in spatial frequency, or " $K$ " space. This concept of K space has begun to play a significant role in radiology since the advent of cross-sectional imaging, and in particular with MRI. A brief digression may serve to make clear the relevance of these ideas to the problem at hand.

Simply stated, to every "normal" space one may pair a $\mathrm{K}$ space, of equal number of dimensions and inverse units of length. For example, to a two dimensional image with length measured in $\mathrm{cm}$, we pair a two dimensional space with length measured in $1 / \mathrm{cm}$. If the coordinates of the image are $(X, Y)$, the coordinates of the $K$ space are $\left(K_{x}, K_{y}\right)$. An image in $(X, Y)$ space is transformed into a unique intensity map in the $\mathrm{K}$ space, given by no other image, by a mathematical computation named after the French mathematician, Fourier. He showed that each image can be abstractly thought of as an interference pattern of waves of intensity variation. These waves are characterized by a direction given by the direction of the $\left(K_{x}, K_{y}\right)$ vector, and a wavelength $\mathrm{L}$ such that $(1 / \mathrm{L})^{2}=\mathrm{K}_{\mathrm{x}}^{2}+\mathrm{K}_{\mathrm{y}}^{2}$. Intensity at a point $\left(K_{x}, K_{y}\right)$ equals the distance from peak to trough of the wave as identified by the $K$ space coordinate values.

Fourier's original abstract concept has a concrete interpretation within the context of MRI. In the presence of a gradient, these waves correspond to the intensity of nuclear MR signals. This is intuitively suggested by examining the units of distance in the raw data, with the help of the gyromagnetic ratio $\gamma$ for hydrogen (Fig 1): T sec) $\times \gamma(1 / \mathrm{sec} /$ Gauss $) \times \mathrm{G}$ (Gauss $/ \mathrm{cm})=\mathrm{K}$ $(1 / \mathrm{cm}) . T$ represents the time variable during the data acquisition of an echo, while $G$ represents the frequency encoding gradient strength during data acquisition. A similar equation holds

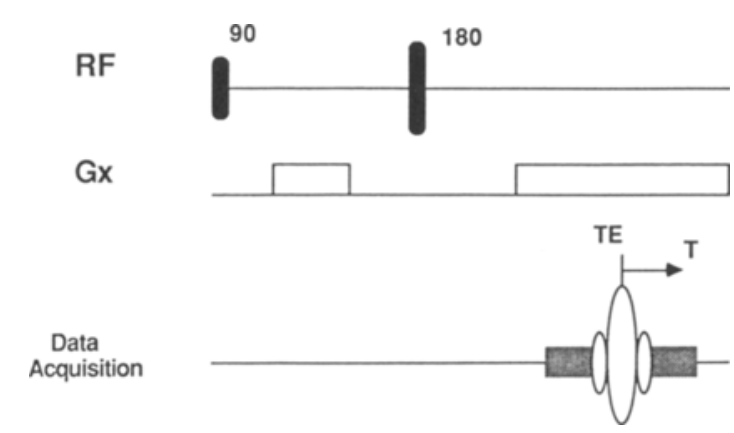

Fig 1. Detection of an echo signal can be thought of as a direct sampling of $K$ space. This can be appreciated with dimensional analysis, which shows that time (sec) is proportional to inverse wavelength $(1 / \mathrm{cm})$.

for the phase encoding gradient, where $T$ represents the length of the phase encoding pulse, a constant, and $G$ represents the height of the pulse, which is the variable from one phase encoding step to another. The dimensional units are the same in either case. In other words, the MR acquisition directly samples $K$ space. The inversion of the Fourier transformation recovers an image from this raw data.

Consider an axial lumbar spine image, with an FOV of $16 \mathrm{~cm}$. The origin in the $K$ space intuitively represents an inverse wavelength of zero, or an infinite wavelength. In the raw data set it occurs at the echo peak of the central phase-encoding step of the raw data, in which the phase encoding gradient equals zero. Intensity at this point in the raw data, or K space, represents the total intensity of the image. The points adjacent to the origin in the digitized raw MRI data, or K space, represent slow oscillations of intensity that go from maximum to minimum to maximum over a distance of one FOV. Intuitively speaking, an image with a given FOV cannot observe true wave oscillations with wavelengths longer than the FOV, and this defines the digital resolution within the $\mathrm{K}$ space. The distance between any two neighboring points in the digitized raw data is therefore $1 / \mathrm{FOV}$, or in this case $1 / 16 \mathrm{~cm}$.

The variation of intensity due to surface coil response, as noted earlier, was typically minimal over a distance of FOV/4. In other words, the surface coil intensity variations are confined to a circle of diameter $4 / \mathrm{FOV}$, or about 4 points, around the origin of the $K$ space. Most of the diagnostically relevant information lies beyond this circle around the origin in the raw data. 
Information on fine detail, edge definition information, and high frequency noise, is contained in points far from the origin of $\mathrm{K}$ space, the points indexing the waves of short wavelength (Fig 2).

One way to obtain a surface coil response map, would be to heavily suppress raw data more than 2 points away from the origin, or central phase encoding echo peak, by multiplying the raw data with a function that decays quickly. Inverse Fourier transformation of this heavily weighted data now gives the desired surface coil correction map. However, this is not the only, nor necessarily the most efficient way to remove the short wavelength components of an image. Digital filtering is a method of preferentially weighting or even deleting information in selected regions of $K$ space without actually transforming the image back into $\mathrm{K}$, or raw data format. A filter that emphasizes the long wavelength components as described above is known as a "low pass" filter because it passes along the low frequency signals, or oscillations, and screens out the high frequency, short wavelength oscillations.

Two major techniques are available for digital filtering, namely the matrix convolution and recursive algorithms. From the image space point of view, low pass filtering blurs an image, reducing the abruptness of intensity variation.
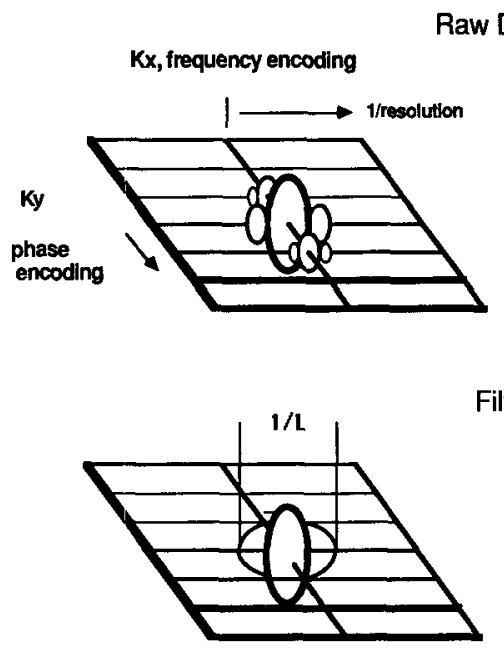

Fig 2. The image has maximum spatial frequency components that contribute intensity to the raw data set out at inverse wavelengths on the order of 1 / (image resolution). By weighting the wavelengths below $1 / L$, the image is blurred, giving an effective resolution of $L$. Although one can do this directly in $K$ space, or the raw data, digital filtering operates on the image date without having to transform in and out of $K$ space.
Intuitively, the intensity of a filtered image at a particular point corresponding to a pixel in the original image can be obtained by simply averaging the intensity over a region of interest (ROI) centered at that pixel. The degree of blurring can be increased by increasing the number of pixels considered to be adjacent to a particular pixel.

The Fourier theory shows that averaging around each pixel in the image with a standard size $R O I$ of radius $L$ is mathematically equivalent to multiplying the raw data by a weighting function that is approximately one within, and drops off quickly outside a radius of $1 / L$ of the origin. In the typical surface coil case, by averaging over an ROI of diameter FOV/4 around each pixel, intensity fluctuations due to anatomic structure will tend to average out, and the net average primarily reflects the local surface coil response. If the ROI is too large (of diameter equal to the FOV) then all averages are approximately equal. This is equivalent to a weighting of $\mathrm{K}$ space of radius $1 / \mathrm{FOV}$, which selects out only the origin. Clearly, for surface coil correction, we wish to make the ROI large, but not too large.

To compute the filtered image, we may use the matrix convolution algorithm that performs this averaging procedure as previously described. The difference between a square ROI and a circular ROI is not important for our purposes. A square ROI is used because it is easier to handle on the computer. Usually the weighting of adjacent pixels is detailed in a square $\mathrm{N} \times \mathrm{N}$ array, or matrix of numbers, giving the name of the algorithm. For an ROI of side length FOV/4 on a $256 \times 256$ pixel image, this is a $64 \times 64$ matrix. Unfortunately, the efficiency of matrix convolution decreases precipitously with an increase in the size of the ROI.

The number of points to be averaged is proportional to the area of the ROI, which increases as the square of the radius of a circular ROI, or the square of the side length of a matrix ROI. A $64 \times$ 64 pixel ROI will require about 4,000 multiplication and addition operations for each of the 65,000 pixels in the image, while a $128 \times 128$ pixel ROI will be four times as lengthy. Smaller ROIs will expand the region of $K$ space that is allowed to contribute to the surface coil response map. This allows anatomic information to leak into the surface coil map, to be subsequently removed from the corrected image. As a practi- 
cal point of reference, the MV4000 computer that supports the General Electric Signa imager at the authors' site required 20 minutes to perform a $30 \times 30$ pixel matrix convolution algorithm on a single image (Fig 3). This ROI is clearly not large enough, even though processing time is prohibitive.

At this point it may not be clear that operating on the image directly has any advantage over operating on the $\mathrm{K}$ space representation. However, more than one numerical strategy is available. In particular, recursive filter theory is a computationally less intense method of implementing low pass digital filters. It can be justified intuitively by noting that the averages over the ROIs of adjacent pixels are very similar, because these ROIs extensively overlap. Figure 4 shows two such pixels and their overlapping ROIs. This figure demonstrates that knowledge of the first pixel's average, and the relatively few pixels not contained in the overlap, is sufficient to calculate the average over the second pixel's ROI. Now that the average is available for the second pixel, it in turn can be used to calculate the average for the next pixel. Such a computation, which intuitively gives the impression of a "snake eating its own tail," is classified as a recursive algorithm, and the interested nonmathematical reader may enjoy a popularized account of the applications of recursion to art, science, philosophy, and literature in an accessible bestseller, Goedel, Escher, Bach. $^{6}$
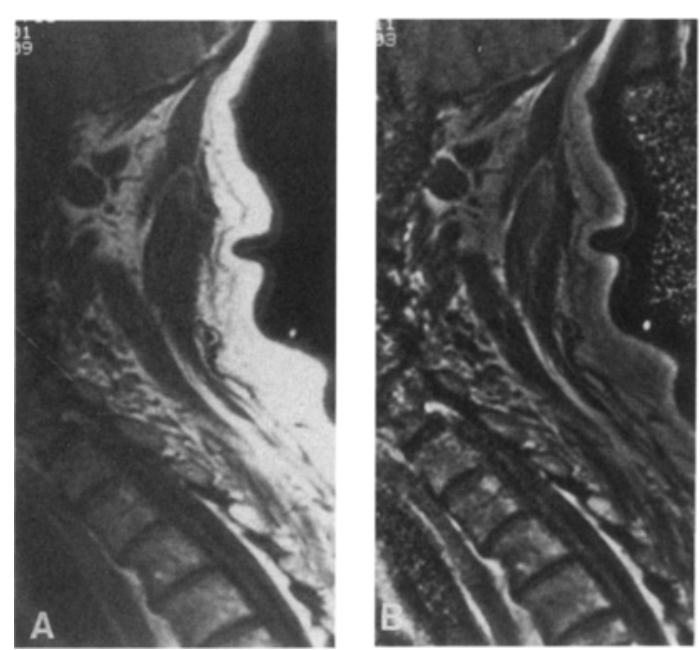

Fig 3. Surface coil correction using matrix convolution: (A) cervical spine exam and (B) "corrected" image.
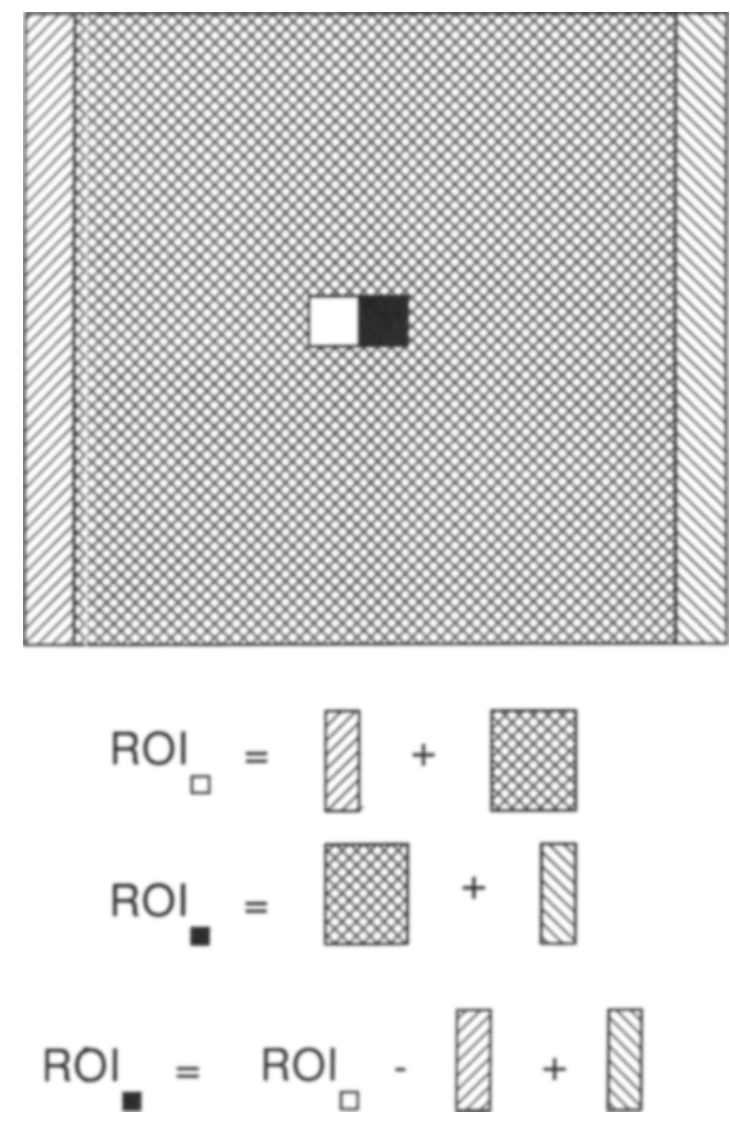

Fig 4. Intuitive explanation of the recursive filter. The average intensity of the ROI centered on the right pixel ( $\square$ ) can be computed from that of the left pixel $(\square)$ by adding and subtracting pixels not in the overlap of the two ROls.
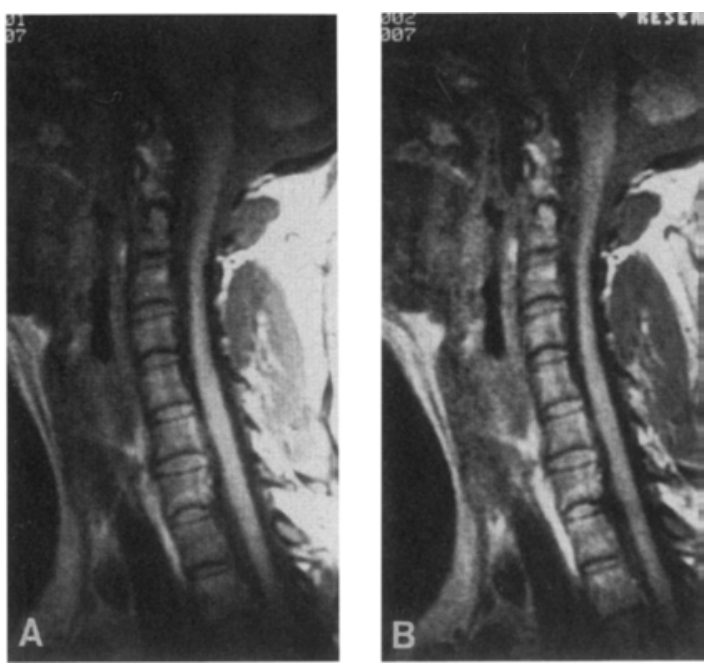

Fig 5. Surface coil correction with a recursive filter: (A) Uncorrected cervical spin exam and (B) corrected image. 
The computation needed to calculate the new ROI average is proportional to the size of the nonoverlapping area, which is about twice the length of the side of the ROI. This computation is much less than that for the matrix convolution, which is proportional to the area of the ROI. Running as a low priority batch process that is available as an option on the TOOLS menu of the Plasma screen of the GE Signa scanner from either the operator or physician console, the algorithm handles ten surface-coil images in approximately ten minutes without severely interfering with normal scanning operations. Alternatively they can be run during off-peak hours and photographed the next morning. A typical series will process in less than $\mathbf{3 0}$ minutes, and is finished in time for the technician to photograph. The corrected images are marked as "research" images. They are appended in a new series to the existing study and can be photographed or archived in the usual manner (Fig 5).

One of the most convenient aspects of this approach is the minimum operator interaction required to correct a set of images. Imaging of a uniform phantom, analogous to a flood phantom in nuclear medicine, is impractical because the surface coil is repositioned with every patient. Theoretical modeling ${ }^{7}$ of the surface-coil fields using the theory of electromagnetism, while ele- gant in principle, will require active prescription of coil position in relation to the image by the technician with every series. In practice, anything that introduces the opportunity for operator error, or reduces available scanning time, is less than optimal in a realistic clinical setting. In comparison to uncorrected images, the filming time is reduced with corrected surface coil images because only one threshold setting is required at filming to capture the entire image.

Note the preservation of normal contrast throughout the corrected image, in this example of herniated lumbar disk disease (Fig 6). Obviously, useful clinical imaging is possible without the use of surface coil correction, as the popularity of surface-coil imaging attests. At the authors' institution, correction has been found to increase confidence in images in which the area of interest extends across the drop-off in surfacecoil response, or in situations where the radiologist wants to make a comparison of tissue contrasts in normal and suspect regions of anatomy (Fig 6). This is especially helpful in situations in which there is widespread soft tissue involvement in pathology, as in Fig 7, a case of osteomyelitis in the thoracic spine.

Because surface-coil correction extends the utility of surface-coil images, it may prove to be even more useful in the future. For example, correction has proved quite useful at our institu-
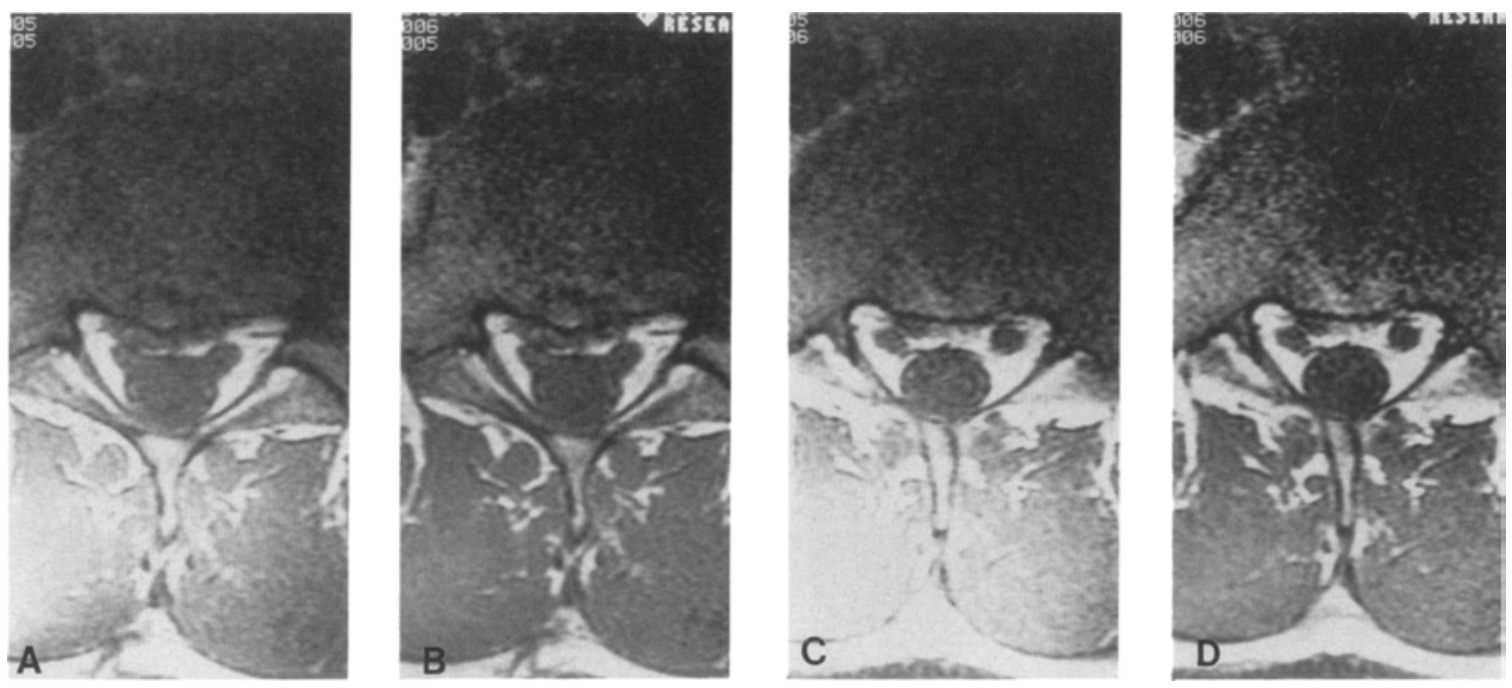

Fig 6. Herniation of the nucleus pulposus, L5-S1. (A) and (C) are uncorrected axial images. (B) and (D) are corrected images. (A) and (B) show disk impinging on the dural sac while (C) and (D) show disk impinging on the $S 1$ root. Note the improved detail in the paraspinous muscles. 

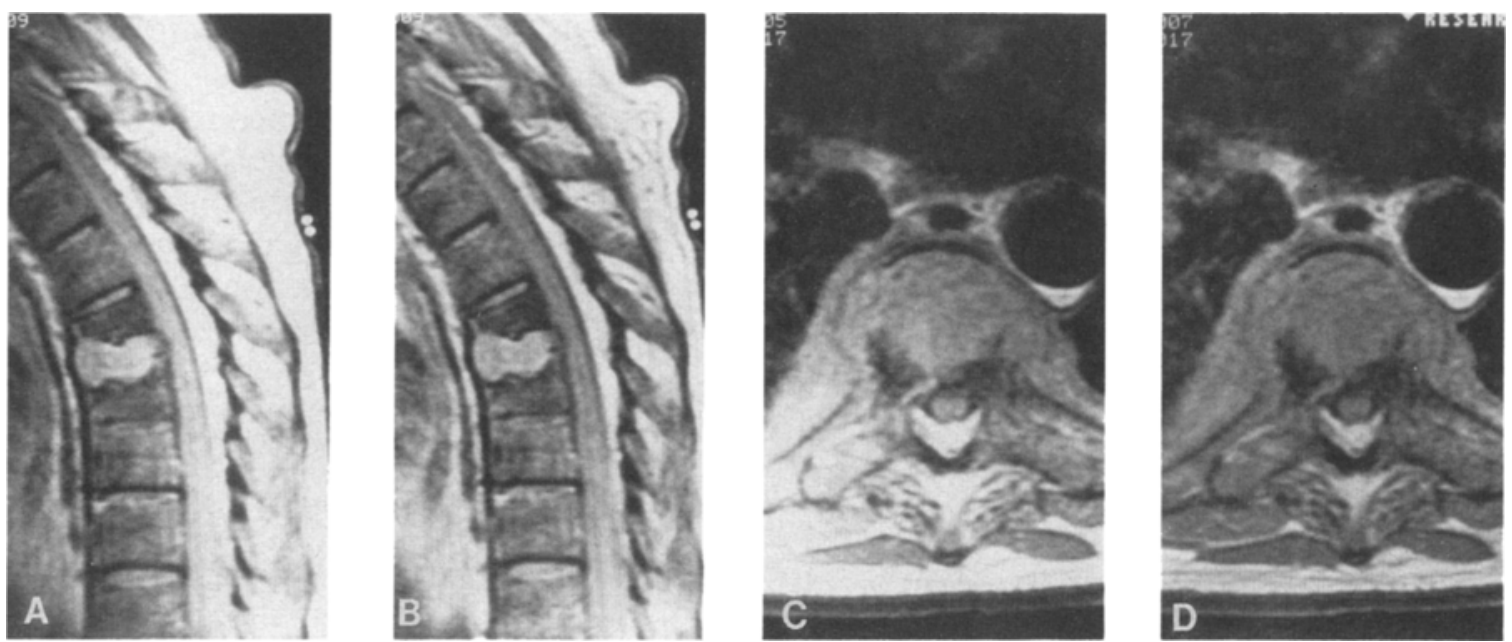

Fig 7. Osteomyelitis of the thoracic spine at T6-T7. (A) and (C) are uncorrected images. (B) and (D) are corrected images. (A) and (B) are sagittal images while (C) and (D) are axial images at the level of the lesion. Note the enhanced appreciation of surrounding soft tissues.

tion for images collected with an experimental endo-rectal coil. The coil lies on the surface of the inflated support, quite close to the rectal mucosa. This exacerbates the effect of surface-coil response drop-off in the uncorrected images, because the coil is only millimeters away from the peripheral zone of the prostate. Figure 8 shows the corrected and uncorrected images of a cancer of the prostate. Preservation of normal contrast in the postprocessed image is essential to distinguish the normal changes in the aging prostate from pathologic changes. Although
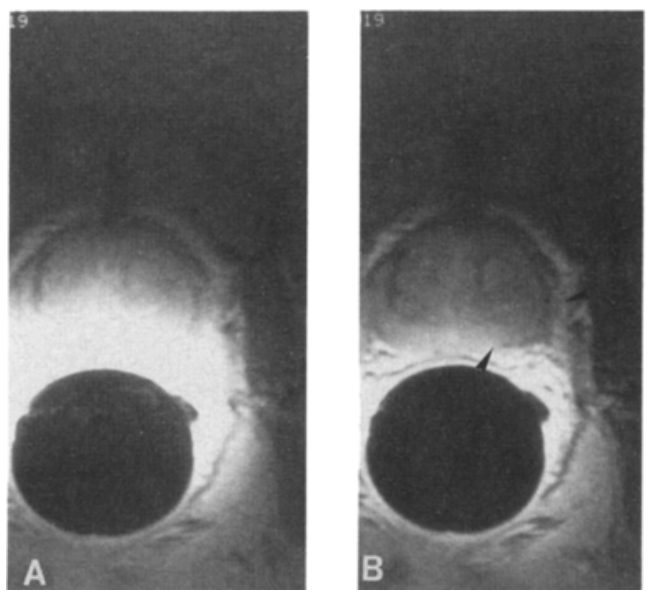

detailed pathologic correlation is ongoing, these images have been read as showing invasion of the neoplasm beyond the prostatic capsule. Surfacecoil correction has become part of our clinical protocol for the exams shown here.

Should surface-coil correction become a standard option, it would be implemented on an array processor, and be considered a reconstruction option, not one of postprocessing. The considerations raised on the efficiency of recursive filtering should apply to the array processor as well. An optimized recursive algorithm should prove
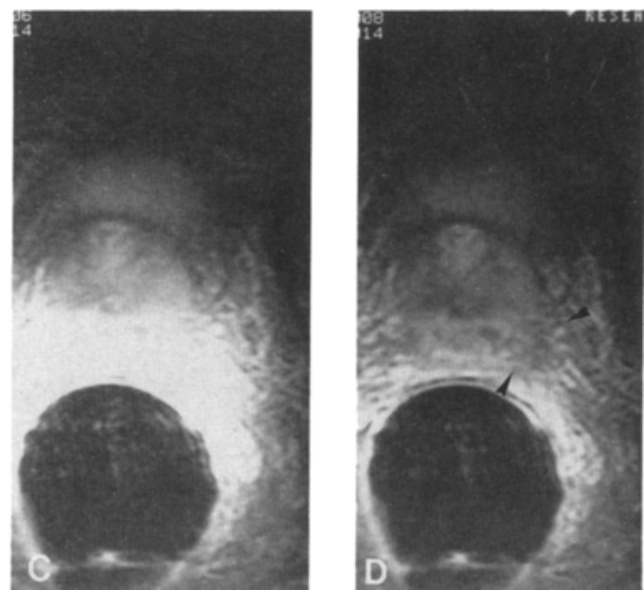

Fig 8. Carcinoma of the prostate. (A) and (C) are uncorrected images. (B) and (D) are corrected images. Experimental surface coil lies on interior surface of the the endo-rectal balloon, unavoidably placing the prostate in the region of significant coil response drop off. Note the improved examination of the peripheral zone of the prostate, showing invasion of the capsule (arrow). 
more efficient on an array processor than either matrix convolution of the image by a large matrix kernel, or two-dimensional Fourier transform of heavily filtered raw data.

Finally, correction algorithms may represent a class of image enhancement techniques that conceptually operate on $\mathrm{K}$ space. The success of such methods depends on isolating an artifact within $\mathrm{K}$ space and selectively deleting this information, while preserving the image information of diagnostic importance. These methods may have other applications in MRI, where image quality is degraded by other factors that contribute to nonuniform shading of the image.

\section{REFERENCES}

1. Axel L, Constantini J, Listerud, J: Intensity correction in surface coil MR imaging. AJR 148:418-420, 1987

2. Haselgrove J, Prammer M: An algorithm for compensation of surface coil images for sensitivity of the surface coil. MRI 4:496-472, 1986

3. Listerud J, Lenkinski RE, Axel L, et al: Surface coil correction. Presented at the Annual Meeting of the Radiologic Society of North America, Chicago, 1988

4. Oppenheimer AV, Schafer RW: Digital Signal Processing. Toronto, Prentice Hall, 1975
5. Pelud A, Liu B: Digital Signal Processing: Theory, Design, and Implementation. New York, Wiley, 1976

6. Hofstadter DR: Goedel, Escher, Bach: An Eternal Golden Braid. New York, Basic, 1979

7. Schenck JF, Hart HR, Jr, Foster TH, et al: High resolution magnetic resonance imaging using surface coils, in Kressel HY (ed): Magnetic Resonance Annual. New York, Raven, 1986 